\title{
Loss as a route to transparency
}

Practical low-loss metamaterials at optical frequencies may soon be realized thanks to optical parametric amplification that uses backwards propagation of a signal beam in negative-index metamaterials. Surprisingly, increasing losses at the idler frequency leads to broadband transparency or amplification at the signal frequency.

\section{Natalia M. Litchinitser ${ }^{1}$ and Vladimir M. Shalaev²}

M etamaterials are extraordinary artificial structures, which can be engineered to exhibit a variety of unique functionalities including a negative index of refraction, subwavelengthresolution imaging, and invisibility and cloaking properties. The majority of the photonic metamaterials realized to date consist of metal-dielectric nanostructures that have highly controllable magnetic and dielectric responses. The problem, however, is that these structures have losses that are difficult to avoid, especially in the visible range of frequencies. The losses originate from a number of sources, including the resonant nature of the metamaterial's magnetic response, intrinsic absorption that is typical of the metallic portion of their constitutive components, and losses from surface roughness. Irrespective of their origin, losses constitute a major hurdle to the practical realization of the unique optical applications of these structures. Therefore, developing efficient loss-compensating techniques is of paramount importance.

Alexander Popov and Sergey Myslivets have now proposed a new approach to overcome losses in metamaterials over the entire negative-index frequency range ${ }^{1}$. The proposed technique is based on a well-known three-wave mixing process that takes place in nonlinear media exhibiting second-order susceptibility (in $\chi^{(2)}$ materials) and induces optical parametric amplification (OPA). However, the unique electrodynamics of negativeindex metamaterials (NIMs) give rise to a rather unusual regime of OPA, making it a promising candidate for converting lossy NIMs into transparent or even amplifying negative-index media.

Optical parametric amplification refers to a process of amplification of a light signal through mixing with pump light in a nonlinear material. Specifically, the photon flux in the signal wave grows through coherent energy transfer from a higherfrequency intense pump wave. A photon from an incident pump laser is divided into two photons, one of which is a photon at the
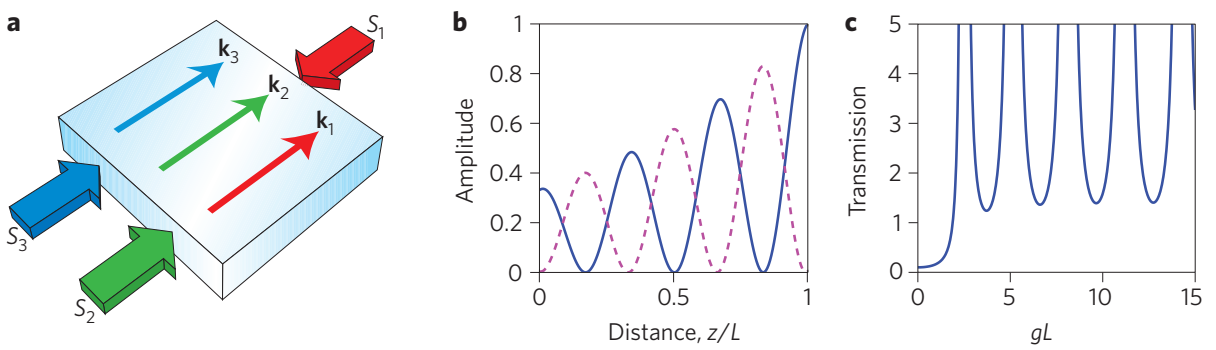

Figure 1 | Optical parametric amplification in metamaterials. a, Schematic representation of backward OPA in a NIM slab. $S_{1}, S_{2}$ and $S_{3}$ are the magnitudes of the Poynting vectors of the signal, idler and pump beam, respectively, and $\mathbf{k}_{1}, \mathbf{k}_{2}$ and $\mathbf{k}_{3}$ are their wavevectors. b. Signal (solid line) and idler (dashed line) field distributions inside the NIM ${ }^{1}$. All waves propagate in the $z$-direction and the signal enters NIM at $z=L$, where $L$ is the slab thickness. c, Signal amplification in the NIM slab. $g$ is a non-linear coupling coefficient ${ }^{1}$. b and $\mathbf{c}$ reproduced with permission from ref. 1; (c) AIP 2008.

signal frequency. The strong pump field with angular frequency and wavenumber $\left(\omega_{3}, k_{3}\right)$ and a weak signal with $\left(\omega_{1}, k_{1}\right)$ generate a difference frequency idler with $\left(\omega_{2}, k_{2}\right)$. The OPA process requires both momentum and energy conservation. The momentum is conserved when the phase-matching condition for the wavevectors, $\mathbf{k}_{\mathbf{3}}=\mathbf{k}_{\mathbf{1}}+\mathbf{k}_{\mathbf{2}}$ is satisfied, whereas energy conservation requires that $\omega_{3}=\omega_{1}+\omega_{2}$.

To date, most OPA schemes have been realized in conventional positive-index materials and are known as forward OPAs, meaning that the energy flows (or Poynting vector, $\mathbf{S}$ ) of all three required waves - the pump, signal and idler - are co-directional. On the other hand, an OPA process involving counter-directed energy flows was predicted to provide significant advantages, including the possibility of optical parametric oscillations without a cavity, compactness and simplicity in both design and alignment ${ }^{2}$. However, in positive-index materials, satisfying the backward phasematching condition is a rather difficult task ${ }^{3}$.

In contrast, metamaterials represent a unique environment for the realization of a backward OPA. Indeed, one of the most fundamental properties of NIMs is that the wave vector, $\mathbf{k}$, (and phase velocity) and the Poynting vector, $\mathbf{S}$, are antiparallel, that is, in opposing directions.
Also, as a metamaterial's properties are frequency-dependent it is possible that the same structure may possess negative-index properties at one frequency but positiveindex properties at another. Thus, an OPA with counter-directed energy flows can be realized with all three waves having co-directed wave vectors, as shown in Fig. 1a. Indeed, if the pump and idler wave propagate in the positive-index regime, and the signal wave frequency belongs to the negative-index range, the energy flow of the signal wave will be antiparallel to that of the pump and the idler (Fig. 1a), creating an effective feedback mechanism ${ }^{4,5}$.

Such a NIM-based OPA was shown theoretically to exhibit peculiar oscillatory distributions of the fields (see Fig. 1b). As a result, the scheme supports oscillations without a cavity as each spatial point serves as a source for the generated wave in the backward direction. Another remarkable property of such a NIM-based OPA, as reported by Popov and Myslivets and illustrated in Fig. 1c, is that the originally lossy NIM becomes transparent (or amplifying) for a broad range of pump field intensities and slab thicknesses, provided that the absorption (loss) coefficient at the idler frequency is equal to or larger than that at the signal frequency. In other words, sufficiently large loss at the idler frequency 
is crucial for the suppression of loss, or amplification, of the signal.

The proposed approach can be compared to another loss-compensating technique explored in the context of NIMs, in which metamaterials are combined with gain media such as optically pumped doped polymers ${ }^{6,7}$. A significant advantage of the OPA approach is that it is not limited to the narrow wavelength range defined by the laser transitions of the laser gain media (for example, rhodamine $6 \mathrm{G}$ or quantum dots), and thus it can be made tunable in a wide frequency range.

Despite the exciting promise of the OPA approach to overcoming loss in a NIM, several fundamental and practical issues are still to be explored. On a fundamental level, the nonlinear optical response of nanostructured metamaterials is not completely understood or characterized, and cannot be predicted precisely. One of the major practical challenges is the realization of bulk nonlinear metamaterials. Other remaining issues include the development of approaches for realizing a controlled absorption coefficient at an idler frequency, which is essential for the proposed scheme; accounting for the pump depletion that is likely to take place in practice; and understanding the noise performance of the proposed device. Nevertheless, backward OPA is a promising route for providing robust transparency and amplification of light in highly absorbing NIMs, and not only has a strong potential to become a practical tool for loss mitigation in NIMs, but also highlights new physics enabled by the unique properties of metamaterials.

Natalia M. Litchinitser ${ }^{1}$ and Vladimir M. Shalaev ${ }^{2}$ are ${ }^{1}$ in the Department of Electrical Engineering, The State University of New York at Buffalo, 309 Bonner Hall, Buffalo, New York 14260-1920, USA; ${ }^{2}$ at the Birck Nanotechnology Center, Purdue University, 1205 West State Street, West Lafayette, Indiana 47907-2057, USA.

e-mail:natashal@buffalo.edu; shalaev@purdue.edu

References

1. Popov, A. K. \& Myslivets, S. A. Appl. Phys. Lett. 93, 191117 (2008)

2. Harris, S. E. Appl. Phys. Lett. 9, 114-116 (1966).

3. Canalias, C. \& Pasiskevicius, V. Nature Photon. 1, 459-462 (2007).

4. Popov, A. K. \& Shalaev, V. M. Opt. Lett. 31, 2169-2171 (2006)

5. Popov, A. K. \& Shalaev, V. M. Appl. Phys. B 84, 131-37 (2006).

6. Klar, T. et al. IEEE J. Quantum Elect. 12, 1106-1115 (2006).

7. Noginov, M. A. et al. Phys. Rev. Lett. 101, 226806 (2008).

\section{ATOMIC MEMORY}

\section{A little nonlinear help}

Storing a light pulse in a vapour is by now a standard laboratory technique. For such optical memory to become truly practical, however, the fidelity of the technique has to be improved. Combining light storage with nonlinear wave mixing may offer a way forwards.

\section{Michael Fleischhauer}

A $\mathrm{n}$ all-optical memory for light signals is a key ingredient for classical as well as quantum information technology, and several techniques have been developed for this purpose over the past few years. One promising approach is based on a phenomenon known as electromagnetically induced transparency

a
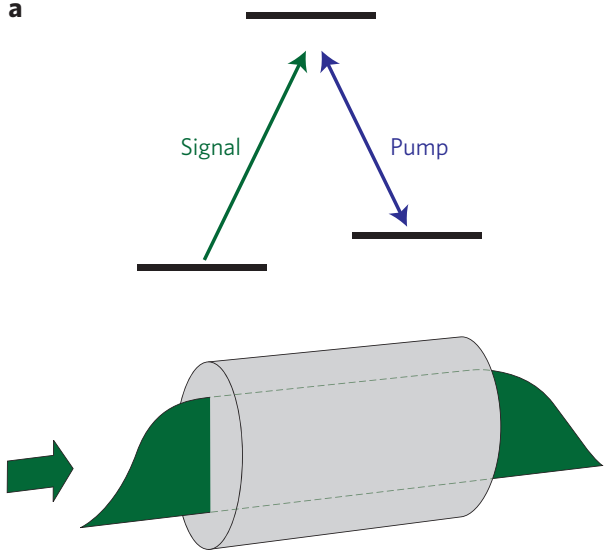

(EIT), where the information carried by an incoming light pulse is transferred to an atomic spin transition by means of a control laser in a resonant Raman process. Although this technique has been demonstrated in vapours, its fidelity - that is, the extent to which original and retrieved pulses agree is far too low for practical applications,

b
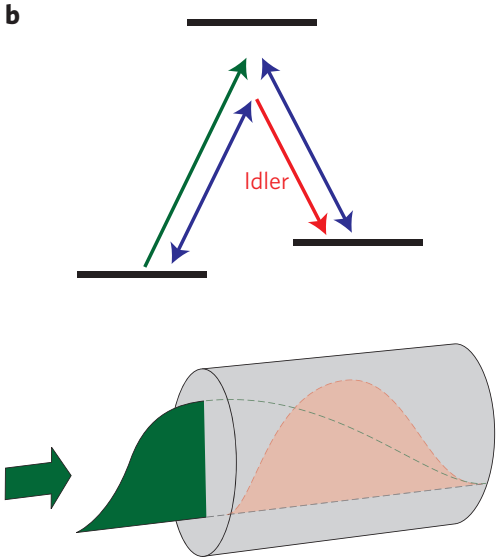

Figure 1 | Light storage assisted by electromagnetically induced transparency. a, Light storage in vapour cells can be achieved by means of EIT where a pump laser (blue) couples to a signal field (green) by a Raman transition to an atomic spin (top). However, the process is often limited by the impossibility of squeezing the entire signal pulse into the vapour cell (bottom). b, Camacho et al. have found an interesting method that may help to circumvent this problem. Light storage accompanied by the nonlinear generation of an additional idler field (red) leads to an effective additional compression, which enables the entire input pulse shape to be extracted upon retrieval.

such as quantum communication and information processing. A recent experiment by Ryan Camacho and colleagues from the University of Rochester and the California Institute of Technology, discussed on page 103 of this issue ${ }^{1}$, suggests that a combination of nonlinear wave mixing and EIT light storage may help to solve this problem.

One of the main challenges of this form of light storage is to capture the entire input light pulse so that it can be transferred to the atomic spin system. Because EIT is based on a resonant phenomenon, the bandwidth of EIT memory is limited to values of the order of the linewidth of the atomic transition, which is typically a couple of tens of megahertz and corresponds to a pulse duration in the microsecond regime. To be able to store such pulses, which are several hundred metres long, their entire length has to be squeezed into the storage medium in one go.

Scientists make use of a relatively simple trick to achieve this. The velocity of the light pulse inside the medium is many orders of magnitude smaller than in vacuum. As a consequence, the pulse is spatially compressed on entering the atomic ensemble: the front end, which is already inside the ensemble, moves with a low velocity, and the back end, which is still propagating at the speed of light in air, quickly catches up. 